\title{
Base-Stock Policies for Two and Three Stages Serial Inventory Systems with Stochastic Demand and Replenishment
}

\author{
D.D. Ntio ${ }^{1^{*}}$, M.J. Vidalis ${ }^{2}$ \\ Department of Business Administration, University of Aegean, Chios island, Greece \\ 12dntio@teikoz.gr, ${ }^{2}$ mvid@ba.aegean.gr, ${ }^{2}$ mvid@ath.forthnet,gr
}

\begin{abstract}
In this paper the impact of pure base-stock replenishment policies on performance of multi-echelon supply chain networks is being examined. Specifically, we try to evaluate cycle inventory, service level, fill rate, work in process, given the replenishment policy. Serial supply chain systems are being studied; such inventory systems receive inventories from only one source and send inventories only to one receiver. The demand and the replenishment process are stochastic. The replenishment processes follow the Erlang distribution. The external demand is distributed by pure Poisson process which means that the amount that each customer asks is one unit. The supply network is modeled as continuous Markov processes with discrete states. The structures of the transition matrices of those systems are explored and computational algorithms are developed to generate them for different values of systems parameters. Mat lab software is used for any computation.
\end{abstract}

Keywords: inventory policies, base-stock policies, multi - echelon supply chain, performance measures

\section{Introduction}

Demand's and supplies' uncertainty leads companies to preserve inventories. Demand is not predictable and constant; it is characterized by seasonality and fluctuations. As far as supplies are concerned, some times it is very difficult for commodities' quality, availability, delivery and transportation time to be scheduled. Thus organizations keep safety stocks in order to deal with uncertainty and satisfy customer needs. Moreover, higher levels of safety stock help companies both to achieve high service level and to avoid the cost of a stock out. On the other hand building up inventories is expensive.

The main objective of every supply system is to cover external demand and satisfy customers with the minimum total cost and maximum profit for enterprises. For this purpose many entities such as producers, manufacturers, suppliers, wholesalers, retailers etc. must cooperate. In real life supply networks include a lot different stages or echelons; the multi echelon supply chains. All those organizations may not have the same goals, each of them are independent decision centers with their own inventory strategy. However, all members of a supply chain realize the benefit of the organization, identify with the whole supply chain's benefit, so they have to coordinate in order to maximize both the total performance of the supply chain and their profits (Chopra \& Meindl, 2007).

This research focus on multi echelon inventory systems, that face uncertain demand and replenishments. Moreover, serial inventory systems with two and three stages have been investigated.

They describe the distribution of finished goods from a manufacturer-producer to a wholesaler and to a retailer. The demand and the replenishment process are stochastic. Orders follow continuous 
review, pure base-stock policies (S, S-1) the ordered quantity is always one unit. When the inventory level drops to $S-1$, an order is placed to increase inventory position to level $\mathrm{S}$. The replenishment processes follow the Erlang distribution. The external demand is distributed by pure Poisson process which means that the amount that each customer asks is one unit. Last but not least the last upstream node is always considered saturated.

The supply networks are modelled as continuous Markov stochastic processes. The structures of the transition matrices of those systems are explored and computational algorithms are developed to generate them for different values of systems parameters (Diamantidis et al, 2004). Mat lab software is used for any computation.

\section{Literature review}

Stochastic inventory systems have been studied since Clark and Scarf in 1960, who provided a technique for finding safety stocks in multi echelon systems with serial topology. Scharz (1981) analyses physical distribution by emphasizing on supply chain performance measures

Base stock inventory policy or one-for-one replenishment policy has its roots in Clark and Scarf's (1960) research about echelon inventory policy for multistage incapacitated inventory systems. Such a policy is described by a single integer parameter $S e \geq 0$, the base stock level (Zipkin, 1991). Base-stock policies are frequently used in situations where order-setup cost is small compared to other costs, such as inventory-holding costs. Due to this policy orders must be placed when the amount of inventory position falls below the base stock level S. In this research each location follows pure base stock policy (S, S-1), thus the order quantity is always one unit.

Base stock policies have been used by manufacturing systems and distribution systems. Rubio and Wein 1996 employ base stock policy for a manufacturing system with multiple products and make-to-stock strategy. Furthermore, Glasserman and Tayur (1994) analyse the stability of a multiechelon production system under base stock policy. On the other hand Ernest and Pyke (1993) examine a two level distribution system with periodic review base stock policy. Also Song and Zipkin (1996) compute performance measures for a two echelon inventory system under base stock policy.

More recently Johansen (2001) and (2005) studies multiechelon systems with continuous review inventory policy in order to find the optimal base stock policy. Finally, Hoberg, Bradley, and Thonemann, (2007) study the effect of various inventory policies and inventory variability, which are key drivers of supply chain performance

\section{Methodology}

The main objective is the performance optimization of multi echelon supply network. Thus a prescriptive model is developed so that an efficient inventory decision process to be formulated. The prescriptive optimization model is based on denotation that $\mathrm{f}(\theta ; \omega)$ is a performance measure depending on a set of decision variables $\theta$, which are under our control and a set of random variables which are beyond our control: the dependence on random events is expressed by $\omega$. (Brandimarte and Zotteri, 2007, p:49). A performance evaluation model aims at estimating the expected value of the selected performance measure:

$$
\mathrm{H}(\theta)=\mathrm{E}_{\omega}[\mathrm{f}(\theta ; \omega)]
$$

Since there is randomness in any supply chain, we actually want to optimize (maximize) the expected value of some performance measure:

$$
\max \mathrm{H}(\theta)=\mathrm{E}_{\omega}[\mathrm{f}(\theta ; \omega)], \theta \varepsilon \Theta
$$

where $\Theta$ is the feasible set for the controlled parameters $\theta$.

In our case the inventory control policies and the replenishment rates are the controllable parameters and the external demand is the uncontrollable parameter. Given a variety of different rates for the controllable parameters and with the background of Markovian theory, various performance measures are being calculated such as fill rate, W.I.P. (Work-inProcess) and cycle time.

Many business problems or procedures can be described by Markov Chain, which stand for modeling uncertainty of adequate real-world dynamic systems. Due to Markovian property the future behavior of the process depends only on the present state of the process and it is not influenced by its past history. A continuous-time Markov chain is a stochastic process 
that is a reductionism of consequence random variables. The process describes a successive transition from one stage or phase to another with the last absorbing stage. Every phase is considered as a state. State transition occurs at every point of time.

The Markov process $\{X(t), t \geq 0\}\{X(t)$, is completely determined by the probability distribution of the initial state $\mathrm{X}_{0}$ and the one-step transition probabilities $\mathrm{p}_{\mathrm{ij}}(\mathrm{dt})$. In applications of Markov chains the art is (Tijms, 2003, $\mathrm{p}: 142)$ :

(a) to choose the state variable(s) such that the Markovian property holds,

(b) to determine the one-step transition probabilities pij .

In simple words, first the state space and the possible number of the states are defined, then the transition matrix is formed. Transition matrix describes the transition probability from one state to another, it's dimension is equal with the number of the possible states. Finally the steady state probability derived from the transition matrix (Vidalis and Papadopoulos, 2001).

In this paper, the continuous-time Markov Chain is used so that the performance of serial supply networks with two and three stages respectively to be examined. It is assumed that external demand is distributed by pure Poisson process, which means that the amount that each customer asks is one unit. The replenishment processes follow the Erlang phase type distribution with $K \geq 2$ phases. Phase type distributions provide a framework for studying Markov process. Erlang distribution depicts a process that must pass from $\mathrm{k}$ number of phases until it reaches the last absorbing phase. Erlang distribution is selected as a research tool over exponential distribution because it offers lower lead time variability, the variability of lead time diminishes as $\mathrm{k}_{1}$ increases. When $\mathrm{k}=1$ is the case of exponential distribution, so k must be up to two. Finally, for mathematical programming model and the rest calculations the mat lab software is used.

\section{The model}

The first supply network under consideration consists of a wholesaler/supplier and a retailer. The second consists of a producer, a wholesaler/supplier and a retailer. The first describes the distribution of finished goods from a supplier to a retailer and the second, the distribution of finished goods from a producer to a wholesaler and to a retailer.

The retailer faces independent Poisson demand, external customers' demand is exactly one unit (pure Poisson) with arrival rate $\lambda$. The replenishment processes follow the Erlang distribution, with replenishment rates $\mu_{1}$ for retailer and $\mu_{2}$ for wholesaler. Orders due to Erlang distribution must pass through $\mathrm{k}_{1}$ and $\mathrm{k}_{2}$ number of phases in order to be delivered to wholesaler and to retailer respectively. Both retailer and wholesaler control inventories with continue review base stock policies $\left(\mathrm{S}_{1}, \mathrm{~S}_{1}\right)$ and $\left(\mathrm{S}_{2}, \mathrm{~S}_{2}\right)$ respectively.

Furthermore, the first upstream node is always assumed to have an infinite capacity, which means

Figure 1. A two stage serial inventory system

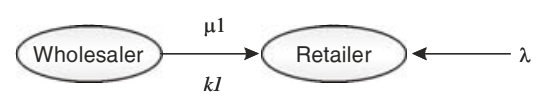

Figure 2. A three stage serial inventory system

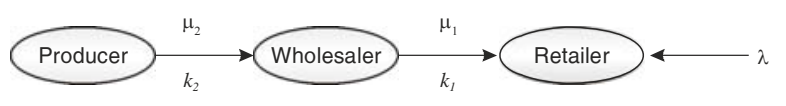

$\lambda \quad$ : demand rate at retailer.

$\mu_{1} \quad$ : replenishment rate from wholesaler the to retailer.

$\mu_{2}$ : replenishment rate from the producer to the wholesaler.

$S_{1} \quad$ : base stock level at retailer.

$S_{1}-1:$ re-order point at retailer.

$S_{2}$ : base stock level at wholesaler.

$S_{2}-1$ : re-order point at wholesaler.

$k_{1}$ : the number of Erlang distribution phases for retailer.

$k_{2} \quad$ : the number of Erlang distribution phases for wholesaler.

that is saturated, always has enough inventory to serve all orders placed by the next downstream node (there are no lost sales). That role in the fist example the wholesaler plays and the producer in the second case. In the second example the wholesaler has finite capacity, thus the retailer's demand it is not always possible to be fulfilled (there are lost sales).

The aim is the calculation of supply chain performance measures such as the fill rate, FR, (the 
fraction of product demand that is satisfied by product in inventory at the retailers) and other performance measures, such as the average inventory, WIP, (the mean number of flow units in the system), and the average flow time or cycle time, $\mathrm{CT}$, (the mean time a flow unit remains in the system), as functions of controllable factors of the system, replenishment rates $\left(\mu_{1}, \mu_{2}\right)$ and inventory policies $\left(S_{1}, S_{1}-1\right),\left(S_{2}, S_{2}-1\right)$.

As it has already been referred the whole model is analysed as a continuous-time Markov process and as a result the performance measures come up after the computation of the possible states of the system, the transition matrix and the steady state probabilities. It follows a simple numerical example for performance measures computation of two stages supply chain network.

The delivery process form a wholesaler to a retailer is being described. The retailer applies a $(3,2)$ continuous review base stock policy, which means the retailer sets an order of one item to a wholesaler when the stock drops to 3-1. It is considered an Erlang distribution with $\mathrm{k}_{1}=2$. First of all, the possible states must be defined. When the system is at a steady state the very next time it may be goes to another, which may be one case of the above:

- To stay to the same condition, there is no phase transition, order-delivery and no customer arrival, which is symbolised by $-\mathrm{k}_{1}{ }^{*} \mu_{1}-\lambda$.

- To transit from one of the $\mathrm{k}_{1}$ Erlang phases to the next and no customer arrival, $\mathrm{k}_{1}^{*} \mu_{1}-\lambda$.

- Order-delivery to the retailer after $\mathrm{k}_{1}=2$ phases and no customer, $\mathrm{k}_{1}^{*} \mu_{1}-\lambda$.

- Customer arrival $\lambda$.

The possible number of the steady states for our example is given by the given function: $S_{1}{ }^{*} k_{1}+1$. To begin with, the steady states must be defined, in the given example for a $(3,2)$ inventory policy there are $3^{*} 2+1=7$ steady states. The steady states of the system (table:1) are codes with two or one digit. The fist number indicates the Erlang phase and the second the retailer's inventory on hand. There are no phases for the inventory position 3 because the retailer doesn't set orders when the inventory is above two unites. For instance, the state 10 denotes that the retailer has zero stock and his order is in the first erlang phase, the 21 that that the retailer has one item in stock and his order is in the second erlang
Table 1. The possible steady states of a two stage inventory system, inventory policy $(3,2)$ and erlang $\mathrm{k}_{1}=2$.

\begin{tabular}{|c|c|c|}
\hline $\begin{array}{c}\text { Erlang Phases } \\
\text { K1 }\end{array}$ & $\begin{array}{c}\text { Retailer's on hand } \\
\text { inventory }\end{array}$ & Steady states \\
\cline { 1 - 1 } 1 & 0 & 10 \\
\cline { 1 - 1 } 2 & 1 & 20 \\
\cline { 1 - 2 } 1 & 2 & 11 \\
\cline { 1 - 1 } 2 & 21 \\
\cline { 1 - 2 } 1 & 3 & 12 \\
\cline { 1 - 2 } 2 & 22 \\
\hline
\end{tabular}

phase one step before the delivery. The 3 steady state, shows that the retailer has three items stock and there is no order in progress. The next step is the transition matrix formulation.

The transition matrix describes the transition probability from one state to another, its dimension equals with the number of the possible states. Thus for $(3,2)$ inventory policy and erlang two, the transition matrix is a $7 \times 7$ table (table:1.1). To be more specific, if the system is in state 3 the very next moment a customer may arrive or there is no customer's arrival, so the system goes to the state 12 or remains to the initial state 3 respectively. If the system is in state 22 in the very next moment a customer may arrive or an order may be delivered or neither a customer arrives or an order is delivered, thus the system transits to the states 21,3 or remain on 22 respectively.

Table 1.1: The transition matrix of a two stage inventory system, inventory policy $(3,2)$ and erlang $k_{1}=2$.

\begin{tabular}{|l|l|l|l|l|l|l|l|}
\hline & 10 & 20 & 11 & 21 & 12 & 22 & 3 \\
\hline 10 & $-2 \mu_{1}$ & $2 \mu_{1}$ & & & & & \\
\hline 20 & & $-2 \mu_{1}$ & $2 \mu_{1}$ & & & & \\
\hline 11 & $\lambda$ & & $-2 \mu_{1}-\lambda$ & $2 \mu_{1}$ & & & \\
\hline 21 & & $\lambda$ & & $-2 \mu_{1}-\lambda$ & $2 \mu_{1}$ & & \\
\hline 12 & & & $\lambda$ & & $-2 \mu_{1}-\lambda$ & $2 \mu_{1}$ & \\
\hline 22 & & & & $\lambda$ & & $-2 \mu_{1}-\lambda$ & $2 \mu_{1}$ \\
\hline 3 & & & & & $\lambda$ & & $-\lambda$ \\
\hline
\end{tabular}


In the case of a three stage inventory system the steady states are defined similarly. The main difference is that the wholesaler sets orders too, so the wholesaler's inventory position and the erlang phases have to be considered as well. As a result the steady state code may be a four digit or three or two digit number. For instance (table: 2), in the case of code 1022 the first digit symbolizes the wholesaler erlang phase, the second the wholesaler's stock, the third the retailer erlang phase and the fourth the retailer's stock. It is being followed the same technique for transition matrix formulation with the two stage case. For transition matrix formulation mathematical algorithms have been developed, by matlab software, for both of two cases. Finally, the steady state probabilities have derived from the transition matrix and performance measures are calculated.

Table 2. The possible steady states of a three stage inventory system, wholesaler's inventory policy $(3,2)$, retailer's inventory policy $(2,1)$ and erlang phases $k_{1}=k_{2}=2$

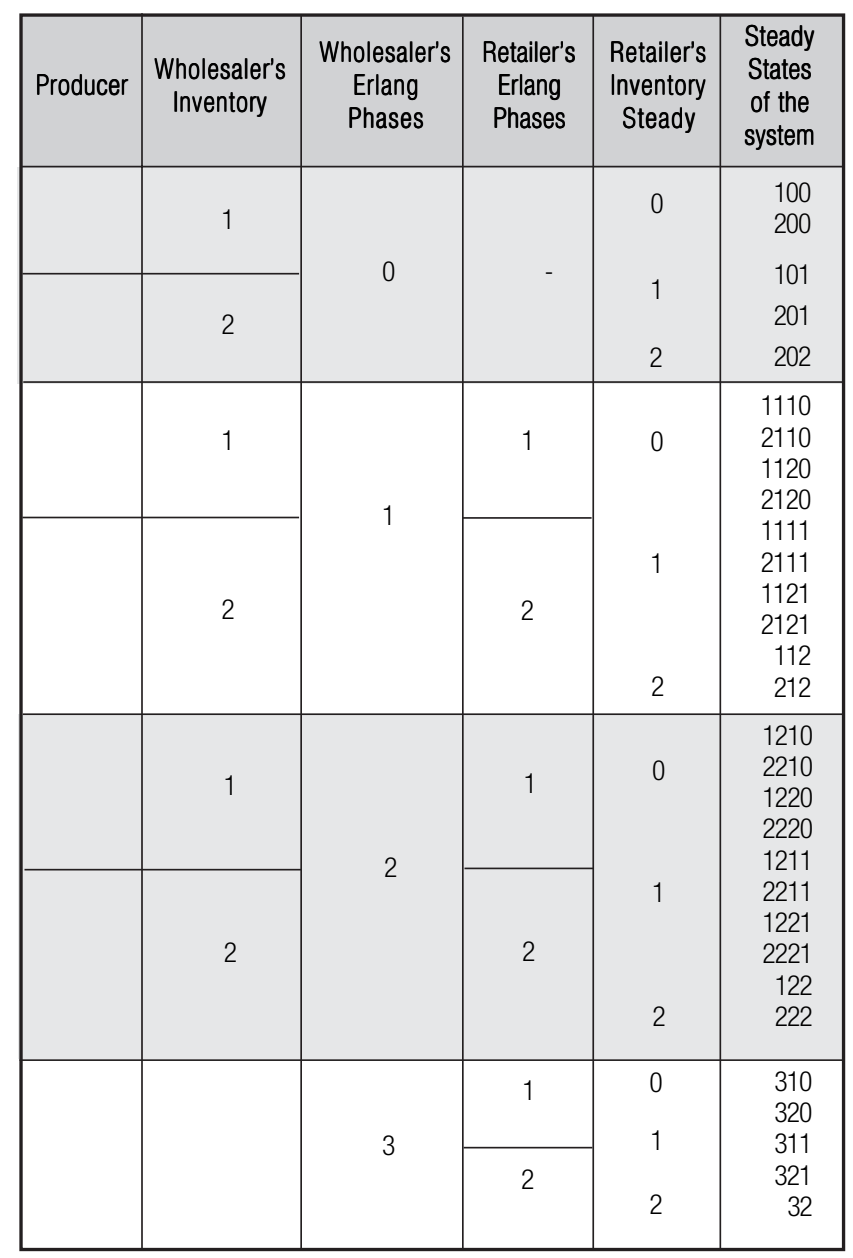

\section{Numerical results}

In this section it is represented some numerical results for the case of two stage serial inventory system. Firstly, the effect of retailer's different inventory policies on the performance measures, it is examined. As the base stock increases, the Fill Rate (figure: 3 ) increases too. There is a positive association between high levels of Fill Rate and high percentages of demand coverage and vice versa. Therefore, in this figure a growing demand fulfilling is depicted. Furthermore, WIP, and Cycle Time rates are increasing when base stock increases. As WIP and Cycle Time rise the holding costs raises. This means that when the base stock is increasing with the other parameters fixed, the demand is better fulfilled however the holding costs are growing.

Table 3. Fill Rate, Wip, Cycle Time for gradually increasing inventory policies, fixed value for $s_{1}=2, \mu_{1}=1, \lambda=1$, and $k_{1}=2$.

\begin{tabular}{|c|c|c|c|}
\hline $\begin{array}{c}(\mathrm{S}, \mathrm{S}-1) \\
\text { Inventory } \\
\text { polycy }\end{array}$ & Fill Rate & Wip & Cycle Time \\
\hline$(2,1)$ & 0,4800 & 1,0000 & 1,4444 \\
$(3,2)$ & 0,7808 & 1,4932 & 1,9123 \\
$(4,3)$ & 0,8302 & 1,9867 & 2,3930 \\
$(5,4)$ & 0,8615 & 2,4819 & 2,8807 \\
$(6,5)$ & 0,8831 & 2,9783 & 3,3725 \\
$(7,6)$ & 0,8989 & 3,4756 & 3,8667 \\
$(8,7)$ & 0,9109 & 3,9736 & 4,3623 \\
$(9,8)$ & 0,9204 & 4,4720 & 4,8590 \\
$(10,9)$ & 0,9280 & 4,9707 & 5,3563 \\
\hline
\end{tabular}

Figure 5. Fill Rate, Wip, Cycle Time for gradually increasing values of $\mathrm{k}_{1}=2-11$, inventory policy $(4,9), \mu_{1}=1$ and $\lambda=1$.

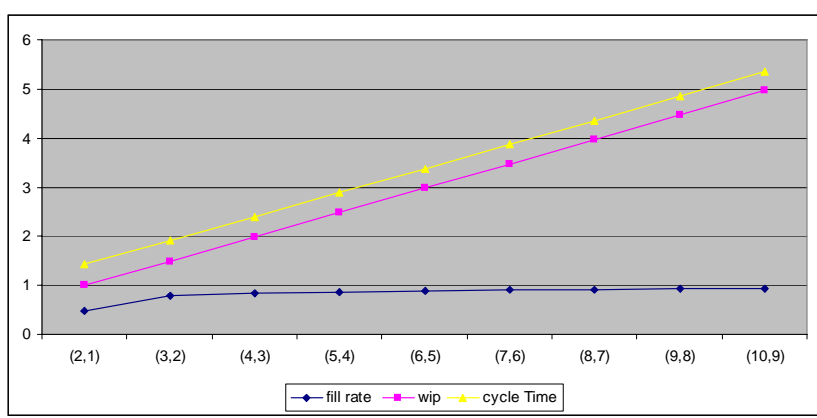




\section{Conclusions and Further Research}

In this research pure base stock inventory policy has been researched in order to expand the management framework for serial inventory supply chains. From the prescriptive model it is derived that as the base stock level and $\mu_{1}$ increase Fill Rate increases. Consequently organizations have the opportunity to meet better the consumers' demand. However, both high rates of $\mu_{1}$ and the base stock level expand the costs of inventory. Enterprises have to make ends meet between the demand satisfaction and inventory cost. The model that has been developed contributes to the inventory decision making by exterminating the proper value for $\mu_{1}$, and the base stock level. In other words it is offering a tool in order to choose between the most appropriate inventory policy which will offer high fractions of demand fulfilling and controllable inventory costs. In the future serial systems with more than three stages could be researched. Therefore, there is a need to develop a mathematical algorithm which will cover all cases.

\section{References}

Brandimarte P. and Zotteri G., (2007), Introduction to Distribution Logistics. Wiley \& Sons, Inc.

Chopra S. and Meindl P., (2007), Supply Chain Management, Strategy, Planning, E Operation, Third Edition. Prentice Hall International, Inc.

Clark A. J., and Scarf H., (1960), Optimal Policies for a multi-echelon inventory problem. Management Science 5, pp. 475-490.

Diamantidis A., Papadopoulos, H. T. and Vidalis M. I. (2004), Exact Analysis of a Discrete Material ThreeStation, Ïne-Âuffer, Merge System with Unreliable Machines. International Journal of Production Research 42 (4), pp. 651-675.
Ernst R. and Pyke D., (1993), Optimal Base Stock Policies and Truck Capacity in a Two-Echelon System. Naval Research Logistics 40, pp. 879-903.

Glasserman P. and Tayur s., (1994), The stability of a capacitated, Multi- Echelon production system under a base stock policy, Operation Research 42 (5).

Hoberg K., Bradley J., and Thonemann W. (2007), Analyzing the effect of the inventory policy on order and inventory variability with linear control theory, European Journal of Operational Research 176 pp: 16201642.

Johansen S., (2001), Pure and modified Base-stock policies for the lost sales inventory system with negligible setup costs and constant lead times. International Journal of Production Economics 71, pp: 391-399.

Johansen S., (2005), Base-stock policies for the lost sales inventory system with Poisson demand and Erlangian lead times. International Journal of Production Economics 93-94, pp: 429-437.

Rubio R. and Wein L, (1996), Setting Bases Stock Levels Using Product- Form Queueing Networks, Management Science $42(2)$.

Schwarz L.B., (1981), Physical Distribution: The Analysis of Inventory and Location. Special ReportProduction and Inventory Control. AIIE Transactions 13(2).

Song J., Zipkin P. (1996), Evaluation of Base-Stock Policies in Multiechelon Inventory Systems with StateDependent Demands. Part II: State-Dependent Depot Policies, Naval Research Logistics 43, pp. 381-396.

Tijms H.C. (2003), A First Course in Stochastic Models, John Wiley \& Sons, Ltd.

Vidalis M. I. and Papadopoulos H. T., (2001), A Recursive Algorithm for Generating the Transition Matrices of Multistation Multiserver Exponential Reliable Queueing Networks, Computers $\mathcal{E}$ Operations Research 28 (9), pp.853-883.

Zipkin P (1991), Evaluation of Base-Stock Policies in Multiechelon Inventory Systems with CompoundPoisson Demands, Naval Research Logistics 38, pp. 397412.

Ms D. Ntio holds a B.Sc. (2002) and a M.Sc. (2004) in Business Administration, from the Aegean University. Nowadays she is a Ph.D. candidate (2008-today) in Supply Chain Management, University of Aegean, Business Administration Department. Since 2005 she has been an adjunct lecturer at Technological Institution of Western Macedonia. She teaches, quality management, communications and advertisement and ERP systems. Her research interests include multiechelon supply chain management, serial inventory systems, replenishment policies with stochastic demand and simulation of supply chain systems. Her research appears in the proceedings of Hellenic Society for Systemic Studies (HSSS), $6^{\text {th }}$ National \& International Conference, $9^{\text {th }}$ Special Conference of the Hellenic Operational Research Society (HELORS) and 1st Olympus International Conference on Supply Chains. 
Dr Michael Vidalis is an assistant Professor of Economics at the Aegean University, Department of Business Administration since 2006. He teaches, at undergraduate level Logistics, Analysis and Modeling of Production-Service Systems and at postgraduate level Logistics, Operations Management, Simulation. He holds Ph.D in Operations Research/Stochastic Modeling University of Aegean, (1998), an MSc OR Computer Science, National University of Athens, (1983), he also has a B.Sc. in Mathematics, National University of Athens, (1978). His research interests include Logistics, Analysis and Modeling of Production-Service Systems, Operations Management, Simulation. He has published two books and over 10 papers in international academic journals. Finally, he has 10 presentations in international conferences and 17 in national conferences. He also is referee of international of Internationals Journals Annals of Operations Research and International Journal of Production Economics. 